\title{
Clustering Learning Model of CCTV Image Pattern for Producing Road Hazard Meteorological Information
}

\author{
1)Jiwan Lee, 1)Bonghee Hong, "1)Sunghoon Jung, and 2)Victor Chang \\ ${ }^{1)}$ Department of Electrical and Computer Engineering, Pusan National University, Busan, South \\ Korea \\ 2)IBSS, Xi'an Jiaotong Liverpool University, Suzhou, China \\ \{wldhks85, bhhong, shjung\}@pusan.ac.kr, Victor.Chang@xjtlu.edu.cn
}

The method of real-time estimation of weather, especially the amount of rainfall, by analyzing CCTV images is much cheaper than one using the existing expensive weather observation equipment. In this paper, we propose a method to find an estimation model function which has its input as CCTV images and output as the amount of rainfall. From the CCTV images, we propose an algorithm for selecting the number and size of the region of interest optimized for rainfall estimation, generating a data pattern graph showing a clear distinction from the number of region of interest, clustering the pattern data graphs, and estimating the amount of rainfall. Experiments using real CCTV images show that the estimation accuracy is over $80 \%$. 


\section{Introduction}

Road hazard weather information such as rainfall, fog visibility, wind speed, snow fall and road icing is very important in achieving sustainability not only for safe driving on road but also for road safety management. To achieve sustainable safe driving on a road, road hazard information as well as traffic information $[1,2,3,4]$ should be provided for vehicle drivers by road section. Road Weather Information System (RWIS), which is installed by road section for collecting road weather information, would be considered to be a weather observation IoT equipment including rainfall, wind direction, wind speed, snow cover sensors.

It is, however, too much expensive to install a large number of loT-based RWISs at regular intervals on road to produce road hazard weather information. Instead, it is much less costly to produce road weather information by analyzing on-line CCTV images installed at regular intervals on existing highways. The purpose of this study is to investigate whether it is possible to produce weather information by using CCTV image which does not require additional installation cost to produce road weather information.

It is the motivation of this paper to investigate whether it is possible to attempt to produce on-line road hazard weather information of the road section by using big data analytics with historical CCTV images of which volume would be huge. The increasing velocity of CCTV video data is considerably greater than the rate of increase in text data. To support veracity that determines the accuracy and validity of the CCTV video data analysis, we need to develop a new estimation learning model that uses historical CCTV video data with RWIS's road weather information. Research on the analysis of CCTV video data has mainly been focused on object identification and multiple object motion pattern $[5,6,7,8]$. As we know, no research has been done to find minute weather changes using CCTV video data.

We develop an estimation learning model by analyzing big data composed of CCTV video image and RWIS observation data. We consider the weather information estimation model as a function $\mathrm{y}=\mathrm{F}(x)$ and consider past CCTV image data as input $\mathrm{x}$ and past weather observation data as output $y$. In order to create a model function $F$, finding the data pattern that can represent fine weather changes from the CCTV image is the key to solving the problem. It is a good measure to make a good estimation learning model by selecting patterns that can find weather change well from CCTV image data to be used as learning data.

The forest background area of CCTV image has been examined whether it best represent the weather change such as the precipitation change. The several background areas in CCTV 
images are forests, roads, and skies. Next, we look for regions of interest $[13,14]$ that are well represented in meteorological changes within a specific background area of CCTV image. The optimal size of the region of interest and the optimal number of regions of interest are examined by experiments on CCTV image data. In this paper, we have found that selecting multiple regions of interest, including not only the near region but also the far region of interest, is the best representation of the minute changes in the image to detect minute changes in the weather. The selection of the best background area and the selection of the region of interest within CCTV image is a main factor that enables clear weather estimation in the CCTV image that best represents minute weather changes.

If the multiple regions of interest are selected for a given CCTV image, the difference pattern of image conversion values between regions of interest is generated. For example, if there are four regions of interest, we create $3 \times 2 \times 1=6$ patterns because we make a difference pattern between all different regions of interest. In the past CCTV images, clustering of image conversion patterns is hierarchically clustering. The original idea of this paper is to express the change of image value according to the change of rainfall with the image transformation pattern cluster. That is, the pattern cluster of CCTV image of rainfall $5 \mathrm{~mm}$ are different when the rainfall is $10 \mathrm{~mm}$. The more variations CCTV images for various rainfall, the higher the accuracy of big data analysis. Therefore, veracity depends on the volume of big data, which has a huge amount of past CCTV video data in which rainfall occurs.

By analyzing big data of CCTV video images in the past precipitation, hierarchical clustering structure that can provide high veracity is created. Next, when the CCTV image for the current time of rainfall is input, a data pattern for the image conversion value is generated. The newly generated data pattern graphs are searched for the hierarchical clusters generated for existing CCTV images by comparing the similarity of generated clusters. The similarity comparison of the data pattern graphs is calculated by the similarity comparison function for the sub-cluster of the hierarchical cluster. The rainfall value is obtained as the label recorded in the sub-cluster with the highest similarity.

The main contributions of this paper can be summarized as follows.

- First, we proposed the best selection of a background area within CCTV video, the multiple regions of interest, the optimal size of the ROI, and the optimal number of ROls that can best represent minute weather changes using the actual CCTV image data. 
- Second, we proposed an algorithm that automatically selects the ROI that best represents the fine weather changes.

- Third, we proposed a method to generate the difference of image conversion value between multiple ROls which best represents the change of the images that best represents fine weather change.

- Finally, we propose a method to generate the data pattern graphs that best represent the difference of image conversion values between multiple ROls, to construct a hierarchically clustered structures for providing an estimation learning model, and to estimate road weather information with similarity difference function.

\section{Road Weather Observation Information and Problem Definition}

This section describes the loT sensing environment for collecting input / output data of the estimation learning model of road weather pattern. The CCTV images on the road side are used as the $x$ values of the model, and the weather observations collected using the loT based sensor are used as the $y$ values.

\subsection{Road Weather Observation Information}

Temperature, humidity, wind, visibility, rainfall, and snowfall are used as loT observation sensors $[9,10,11,12]$. A Road weather observation equipment to install these weather observation sensors on a specific pole is called stationary RWIS. Mobile RWIS is to install meteorological equipment on a moving vehicle to collect meteorological information for verification of CCTV images far from the stationary RWIS.

The road weather observation data from stationary RWIS and mobile RWIS are transmitted to the server at intervals of 60 seconds using LTE wireless communication network. Stationary RWIS and mobile RWIS consist of sensor device, data logger, calculation module, and transmission module. The sensor device is a device capable of collecting road weather data such as precipitation, temperature, humidity, snowfall and so on. The data logger accumulates and stores observation data for a certain period of time. The computation module performs an aggregation query to verify the data quality, such as the sum, maximum, and minimum values of the sensor values. Finally, the transmission module transmits the observation data to the server.

In the data processing server, the road weather observation data received from RWIS is 
mapped to specific time zone and road segment. All of the road weather observation data is stored for each road section in the form of a table. The road weather information produced by mobile RWIS, which varies according to vehicle movement, produces sparse road weather information in time and space. Since the stationary RWIS is fixed in position, regular road weather information is produced in time, but there is no weather information value in other road sections.

In Fig. 1, the CCTV location is installed and operated at regular intervals, but it may be different from the link position according to the node-link structure which is the standard of road traffic information. Sampling of CCTV video images is required for extracting video every 15 minutes because the size of the entire video is too large to store in the server. The extracted CCTV video is transmitted to the road management server through the private network. We employed the sampled CCTV video for 60 seconds video frame every 15 minutes.

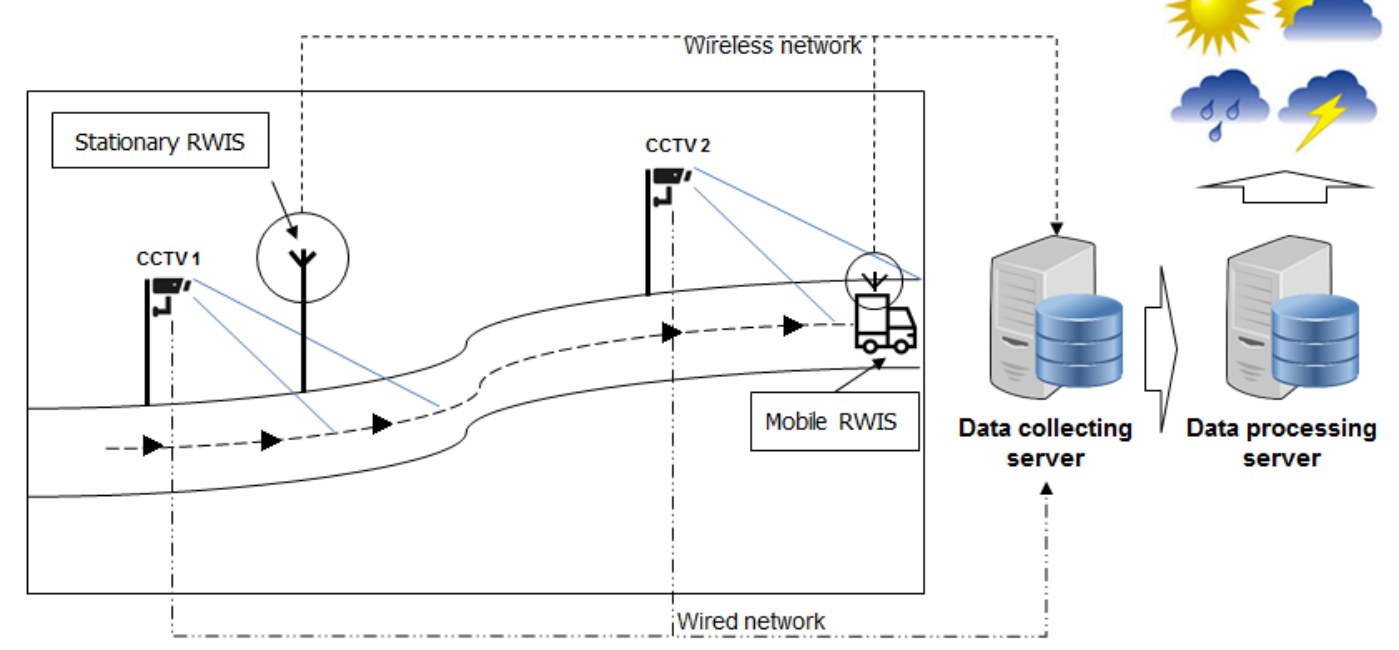

* RWIS: Road Weather Information System

\section{Figure 1 lot observation configuration diagram for real-time collection of road weather} information

\subsection{Data Specification}

The road construction management company stores and manages the CCTV images information on the server device for at least one month in case of an accident on the highway. CCTV video is a set of consecutive frames. The CCTV videos are set of frames, it is expressed as $\left\{\right.$ frame $_{i}$, frame $_{i+1}$, frame $_{i+2}, \ldots$, frame $\left._{j}\right\}$ (where $i$ is the start time of the 
video, and $\mathrm{j}$ is the end time of the video) as shown Fig. 2-(a). Assume that there is a CCTV recording 24 frames per second. If you record CCTV video for 15 minutes, 21,600 frames will be stored in 15 (minutes) $\times 60$ (seconds) $\times 24$ (frames).

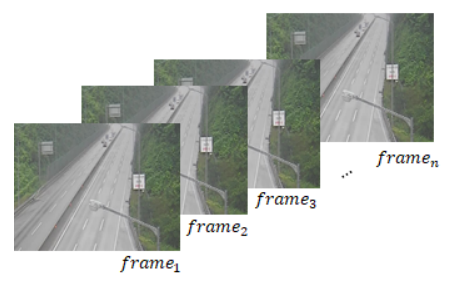

(a)the frame of video

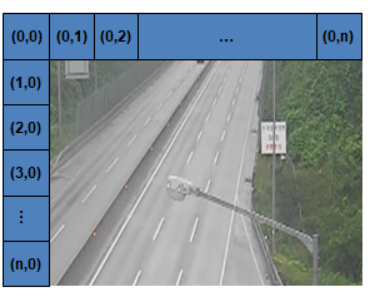

(b)the pixels of a frame
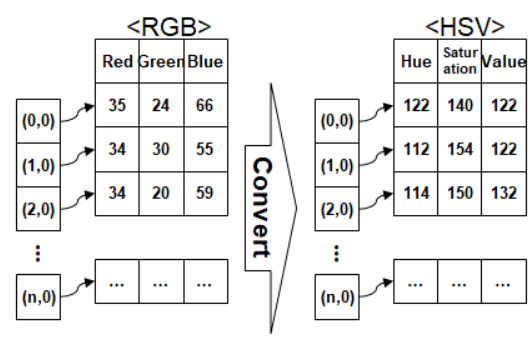

(c) Image converting RGB to HSV

Figure 2. CCTV Video information

Each frame consists of a set of pixels $p_{i j}$, and the specific frame frame $_{k}$ represented by $\left\{p_{00}, p_{01}, p_{02}, \ldots, p_{n m}\right\}$, where $n$ is size of width, $m$ is size of height. The $\mathrm{n}$ and $\mathrm{m}$ of the frame are determined by the CCTV device resolution. Assuming that the CCTV resolution is $1280 \times 720$, the number of pixels is 921,600 . Fig. 2 -(b) shows the rule that the pixel is located on the frame. Suppose that $p_{i j}$ expresses $(0,0)$ pixel. $(0,1)$ pixel that move one space to the right can be expressed as $p_{i j+1}$.

In general, each pixel of an image frame basically has a color value represented by red $(R)$, green $(G)$, and blue $(B)$ color spaces. In addition to $R G B$, there are various color spaces. In this paper, we have used a HSV color space. The color space conversion library was provided by OpenCV [15]. The corresponding library converts RGB values of each pixel into the HSV color space by inputting them as function parameters. Fig. 2-(c) shows the conversion from the RGB color space to the HSV color space.

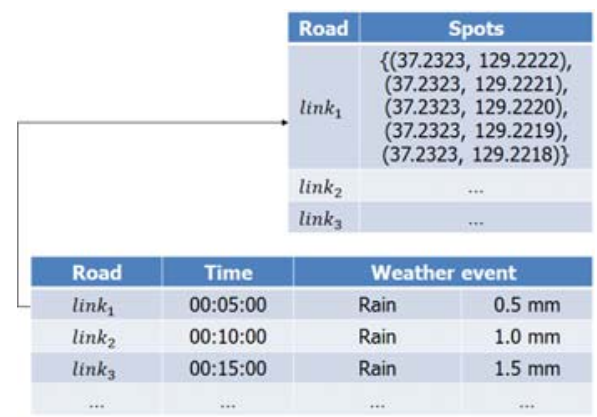

Figure 3. An example of data structure for road weather information

The road weather information represents weather information for each road section. 
Typically, the meteorological equipment produces point-by-point meteorological information, and the area within a certain radius is assumed to be the same meteorological information. This paper assumes that roads consist of nodes and links in order to produce weather information for each road section. As shown in Fig. 3, the road weather information consists of three fields: space, time, and weather. In the field representing the space, you can see that a specific road such as $\operatorname{link}_{1}$ is expressed instead of the administrative area. link $_{1}$ is $\left\{N_{1}, N_{2}, N_{3}, \ldots, N_{n}\right\}$, and each element $N_{n}$ consists of latitude and longitude twodimensional values. In the field representing time, $t_{1}, t_{2}$ and $t_{3}$ are used to represent a specific time. In Fig. 3, let's take an example of the first line $\left(\right.$ link $_{1}, t_{1}$, rain $\left.-1.0 \mathrm{~mm}\right)$. This example means "there was a cumulative precipitation of $1.0 \mathrm{~mm}$ for $t_{1}$ at time $t_{1-m}$ on a particular $\operatorname{link}_{1} "$, where $\mathrm{m}$ is the time interval of the observations set in the road weather instrument.

\subsection{Problem Definition}

In this paper, we develop a non-deterministic model, $y=f(x)$, that generates road weather information with y values using CCTV image information as inputs. There are two approaches to solve this problem. First, it is a method to determine the rainfall amount based on the threshold value of the conversion value for the CCTV image. However, this method is not suitable because it shows the same rainfall even though it is different image as shown in Fig. 4 shows an example of CCTV images appearing differently despite the same rainfall of $5 \mathrm{~mm}$ for the same location. In Figure 4, Fig. 4- (a) and Fig. 4- (b) show the same precipitation, but Fig. 4- (a) shows a slightly lighter color tone than Fig. 4- (b).

CCTV images may not be the same when the same amount of rainfall occurs at the same time in different places. Therefore, it is impossible to determine the amount of rainfall by CCTV image value because there are many cases. Instead, we propose a method of extracting data patterns of past CCTV images for the same amount of rainfall and storing them as learning data. In summary, it is the key idea of this paper to find a non-deterministic model $y=f(x)$ as a weather estimation function when we consider the data patterns extracted from past CCTV images as $x$ values and the rainfall as $y$ values. 


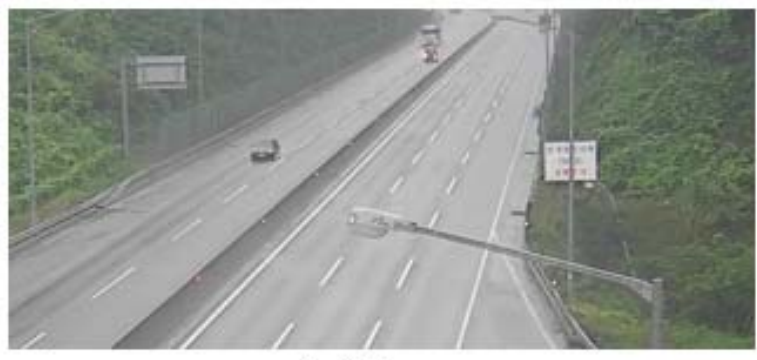

(a) $5 \mathrm{~mm}$

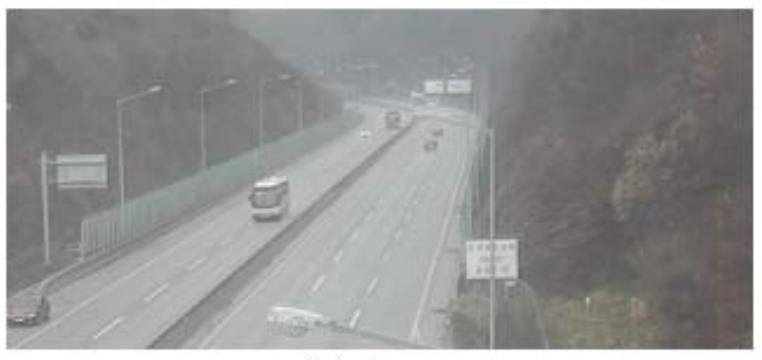

(b) $5 \mathrm{~mm}$

Figure 4. Example of images for the same region, same precipitation

We present three methods to extract the most effective data patterns from the past CCTV images that can represent fine weather changes such as rainfall changes. First, it is necessary to select the most effective background area within CCTV images that can best represent minute weather changes from CCTV images. Most CCTV images have usually three potential target areas: forest, road, and sky. The problem of selecting the most effective background area in the CCTV image is discussed in Subsection 3.1. Second, the region of interest (ROI) should be selected to determine the intensity of the rainfall using the image feature in the selected background area. The reason for selecting the region of interest is that selecting a specific area that best represents the fine weather changes is more representative of weather changes than using the entire CCTV area. The problem of selecting the optimal size and optimal number of ROls is discussed in Subsection 3.1. Third, a method of generating data patterns using multiple ROIs is described in Section 4 , which defines the difference of image conversion values between different ROIs as a pattern. Below table 1 summarizes the meaning of the symbols used in this section.

\section{Table 1. Symbol Description}

\begin{tabular}{c|l}
\hline Symbol & \multicolumn{1}{c}{ Description } \\
\hline \hline$t$ & A specific time \\
$t_{i j}$ & The time from $t_{i}$ to $t_{j},\left\{t_{n} \mid i \leq n \leq j\right\}$ \\
$s$ & A specific space \\
$c$ & A weather condition \\
$c v_{t_{i j}}^{s}$ & A CCTV video located at s from $t_{i}$ to $t_{j}$ \\
$p_{i j}$ & Pixel value of i-th column and the j-th row, $p_{i j} \in \mathrm{P}$ \\
${ }_{c} p_{(t, l)}^{i}$ & a set of pixels on region of $i$ in the CCTV image of given time $t$ and \\
$d p_{(t, l)}$ & location / that satisfies \\
& A data pattern extracted from image on link $i$ at given time $t$
\end{tabular}




\begin{tabular}{|c|c|}
\hline$S F_{c}\left(d p_{(t, l)}\right)$ & A function that returns the number of patterns similar to the pattern of \\
\hline $\mathrm{WI}\left(\operatorname{link}_{i}, \mathrm{t}\right)$ & $\begin{array}{l}\text { A function that returns the digitized road weather information on } \\
\left.\text { specific } \operatorname{link}_{i} \text { at given time } t \text { (ex, rain }-0.5 \mathrm{~mm}\right)\end{array}$ \\
\hline HRWI & A set of historical road weather information \\
\hline$r w i_{(t, l)}$ & A road weather information on specific link / at given time $t$ \\
\hline
\end{tabular}

Problem of selecting target area. Given a time $t$ and a specific link $l$, the problem of choosing the background area of a given CCTV image which can best represent the fine weather change is explained. best background area in a specific image is selected by $\operatorname{MAX}\left(\Delta d_{i}\right)$. The $\Delta d_{i}$ can be expressed as the following equation (1) by obtaining the total amount of change of the image conversion values of the corresponding background area i according to the change in the weather. ${ }_{c} p_{(t, l)}^{i}$ is a set of i-region pixels in a CCTV image that satisfies a given space-time $t, l$, and weather condition c. Fig. 5 shows forest and road area information and corresponding image feature values. In the example, the most suitable analysis area is the "Forest1" area with the highest change according to the weather.

$$
\Delta d_{i}=F_{v}\left({ }_{c} p_{(t, l)}^{i}, \quad{ }_{c} p_{(t, l)}^{i}\right), \text { where i are forest, road, sky. }
$$
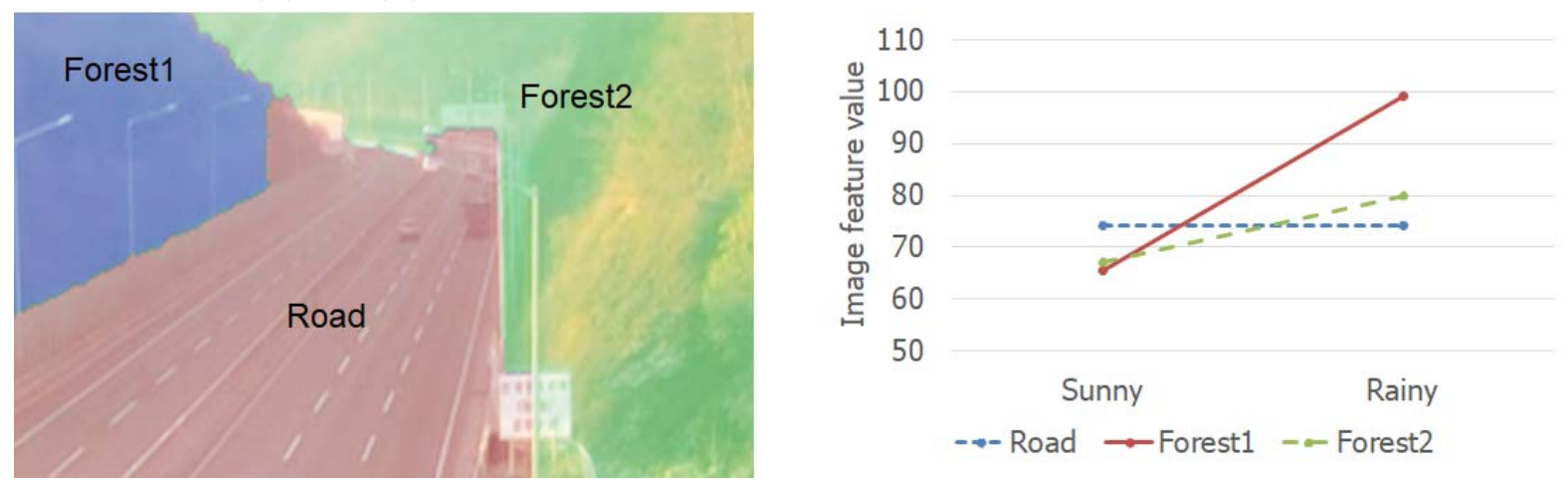

\section{Figure 5 Comparison of image feature values for different background areas}

\section{Problem of selecting best multiple ROI for determining degree of precipitation. The}

set of pixels with a given set of ROls and precipitation intensity ${ }_{c} p_{(t, l)}^{i}$, finds optimal multiple ROls that distinguish the intensity of precipitation. The optimal multiple ROIs are determined by $\Delta v p_{R O I}$. The $\Delta v p_{R O I}$ can be expressed by Equation (2) by obtaining the sum of the changes of $R O I$ region according to the precipitation intensity. The $\operatorname{roi}_{n}$ is an element of $R O I$, it consists of two coordinates as upper left and right bottom. The set of pixels contained in $\operatorname{roi}_{n}$ is referred to as $p r_{n}$, and $\{p \mid p$ is located in rectangle of $\left.\operatorname{roi}_{n}\right\}$. Experiments should be performed while changing the size and spacing of the roi $i_{n}$ 
to find optimal ROls.

$\Delta v p_{R O I}=\sum_{i=1}^{n} F_{v}\left(\operatorname{rain}_{(t, l)} p^{p r_{i}} \quad \operatorname{rain} 1+i_{i} p_{(t, l)}^{p r_{i}}\right)$

where $\mathrm{n}$ is the number of elements in the ROI .

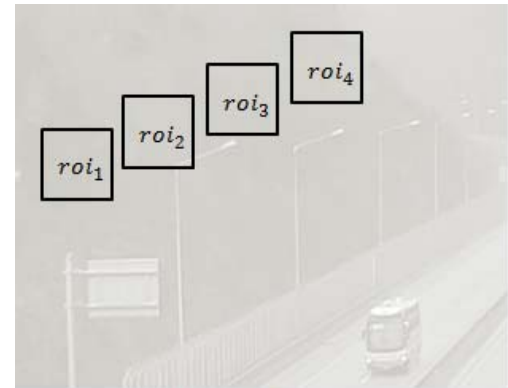

(a) Standard ROI

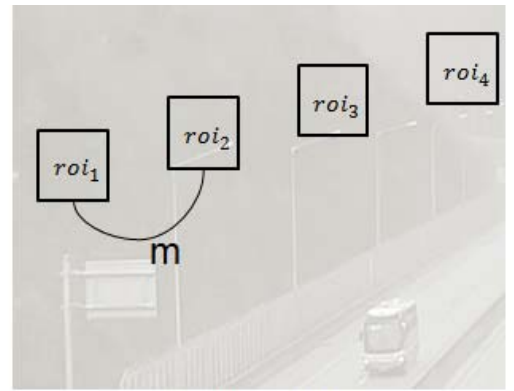

(b) ROI Increased $\mathrm{m}$

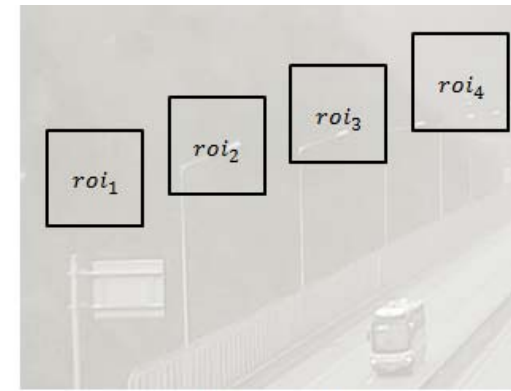

(c) ROI Increased size

Figure 6. An example of roi $_{n}$ by interval and size

Problem of generating numerical road weather information. Given a time $t$ and a specific link $l$, weather information $\operatorname{WI}(l, \mathrm{t})$ is generated if any $\mathrm{t}, \mathrm{l}$ exist where $S F_{c}\left(d p_{(t, l)}\right)$ $>=1$ and $r w i_{(t, l)} \subset$ HRWI. The $S F_{c}\left(d p_{(t, l)}\right)$ function is calculated by comparing the cluster result of the clustering learning model with $d p_{(t, l)}$. In this paper, we develop an weather estimation algorithm based on clustering learning model.

This paper proposes an algorithm to generate road weather information by clustering learning model of big data of existing CCTV images after generating a data pattern graph for CCTV images at the place where rainfall occurred. The following Sections 3 and 4 describe our proposed solution when addressing these problems. 


\section{Multiple ROI selection to best detect weather changes}

In this section, we propose a method for selecting multiple ROIs which are suitable for detecting weather changes. The method is comprised of two steps; First, a background area that can best express weather change is selected by image segmentation $[15,16]$ in the first step. Then, multiple ROIs by distance are automatically selected within the target area in the second step.

\subsection{Selection of Analysis Target Area}

In this section, we explain a method for selecting the best background area in CCTV images, which has distinct feature changes by fine weather changes. As shown in Fig. 7(a), most of images I from CCTV for road management can be divided largely into two regions: road $I_{r}$ and surroundings $I_{s} . I_{,} I_{r}$, and $I_{s}$ have conditions as Equation (3).

$I=I_{r} \cup I_{s}, I_{r} \cap I_{s}=\varnothing$

We apply Watershed[16], one of the image segmentation methods, to segment image regions. Generally $I_{S}$ can be divided into several regions such as forests and sky.

According to the number of markers which are manually assigned for Watershed, the number of regions dividing $I_{s}$ is determined. When we assign $n$ markers for segmentation, one for road and the others for surroundings, $I_{s}$ is consist of $n-1$ regions as Equation (4).

$$
\begin{aligned}
& I_{s}=\bigcup_{i=0}^{n-2} I_{s_{i}} \\
& \forall_{i, j} I_{s_{i}} \cap I_{s_{j}}=\emptyset, 0 \leq i, j<n-1, i \neq j
\end{aligned}
$$

Fig. 7-(b) shows an example of segmented $I_{r}$ and $I_{s}$ by superimposing different colors. 


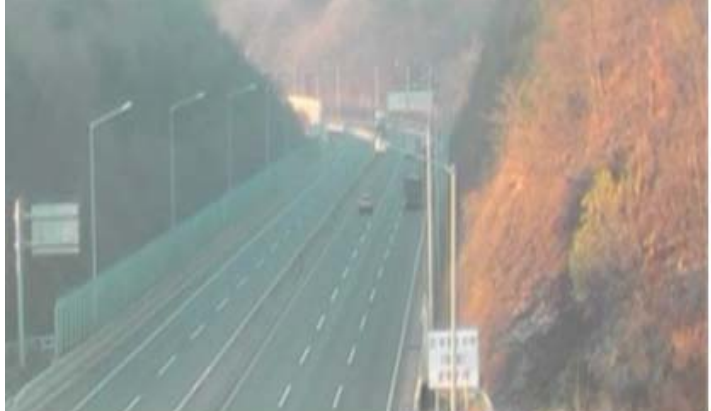

(a) Original CCTV images

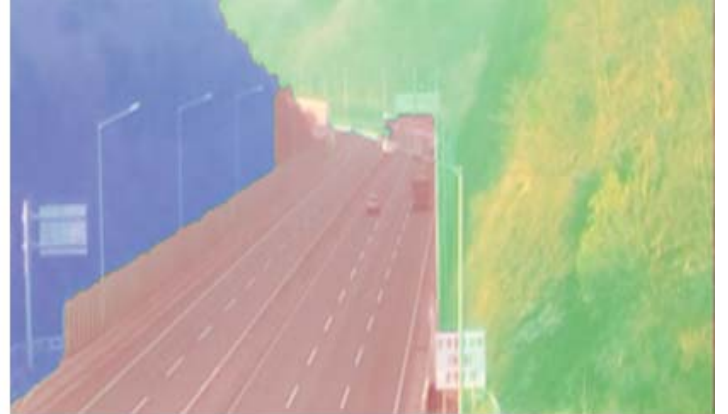

(b) CCTV image after segmentation

Figure 7 Example of division of possible background area by using images segmentation

Assume that left forest region $I_{s_{k}}$ in Fig. 7-(b) is selected as a target background $I_{t}$. As shown in the Fig. 7-(b), $I_{t}$ can include not only forest region $I_{f}$ but also non-forest regions $I_{n f}$, such as traffic signs or street lamps, which are out of our interest. To extract $I_{f}$ from $I_{t}$, we fit the pixels in $I_{t}$ to Gaussian mixture model by using expectation maximization. For each pixel in $I_{t}$, the pixel is classified as non-forest when the Gaussian distribution it falls has weight below a threshold or the probability in the Gaussian distribution it falls is below a threshold.

\subsection{Selection of Multiple ROI}

Applying multiple ROls by distance is more effective than using a single ROI for detecting a pattern of fine weather changes such as rainfall. Thus we devise how to determine best location of ROls.

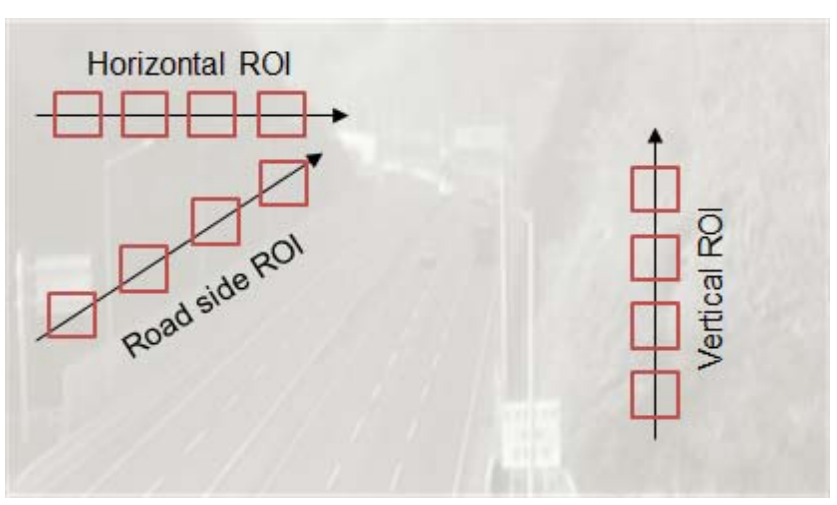

Figure 8 An approach for selecting multiple ROIs

We can consider some ways, such as horizontal, vertical and roadside, to align multiple ROIs on the forest region segmented through Section 3.1. In horizontal case, there is no difference in the distance between ROls, which is inconsistent with the purpose to select multiple ROls. As shown in Fig. 8, in vertical case, the higher ROI located in the CCTV image the farther region data may be acquired. It may not be possible, however, depending on 
the appearance of the forest. In the worst case, regions with the same distance but different height can be selected. In roadside case, it is usually possible to select multiple ROIs by distance from CCTV.

To select multiple ROIs in the target area along the roadside, we determine a guide line GL which passes the vanishing point of the lines extracted from the road in the CCTV image[16, 17, 18, 19]. In real world, lines on the road, such as lanes or median strip, are actually all parallel. When these lines are projected on the image, each line, denoted by $\mathbf{l}_{i}=\left[\begin{array}{lll}a_{i} & b_{i} & c_{i}\end{array}\right]^{T}$, intersects at a point called vanishing point due to projective transform. In real case, however, the cross points of each line pair are not identical due to some errors. Thus we calculate the vanishing point vp by averaging the cross points as Equation (5).

$\mathbf{P}=\left\{\mathbf{p} \mid \forall_{i, j}\right.$ intersect $\left.\left(\mathbf{l}_{i}, \mathbf{l}_{j}\right), i \neq j\right\}$

$\mathbf{v p}=\frac{\sum_{i=0}^{n-1} \mathbf{p}_{i}}{n}$, where $n=|\mathbf{P}|$

The guide line GL is determined by following three conditions: 1) passing by the calculated vanishing point, 2) the slope between 0 and outermost $\mathbf{l}_{\text {out }}, 3$ ) maximal overlap with the target region[20]. The candidate line set $C L$ satisfying condition 1) and 2) is defined as Eq. (6).

$C L=\left\{\left[\begin{array}{l}a \\ b \\ c\end{array}\right] I\left[\begin{array}{l}a \\ b \\ c\end{array}\right] \cdot\left[\begin{array}{c}\mathbf{v p} \\ 1\end{array}\right]=0, \min \left(0,-\frac{a_{\text {out }}}{b_{\text {out }}}\right) \leq-\frac{a}{b} \leq \max \left(0,-\frac{a_{\text {out }}}{b_{\text {out }}}\right)\right\}$

Finally, GL satisfying condition 3) among $C L$ is determined as Eq. (7).

$\forall_{i} \mathbf{C L}_{i} \in C L, O_{i}=\left|\left\{\mathbf{p}_{f} \mid \mathbf{C L}_{i} \cdot\left[\begin{array}{c}\mathbf{p}_{f} \\ 1\end{array}\right]=0, \mathbf{p}_{f} \in I_{f}\right\}\right|$

$\mathbf{G L}=\boldsymbol{C} \boldsymbol{L}_{i}$, where $i=\arg _{i} \max O_{i}$

Multiple ROls are selected by moving from near to far along the determined GL. If nonforest area within the $\mathrm{ROI}$ is above a certain ratio in the process, it is excluded from $\mathrm{ROI}$ selection. In Fig. 9, blue line represents the guide line and blue rectangles represent selected multiple ROls. 


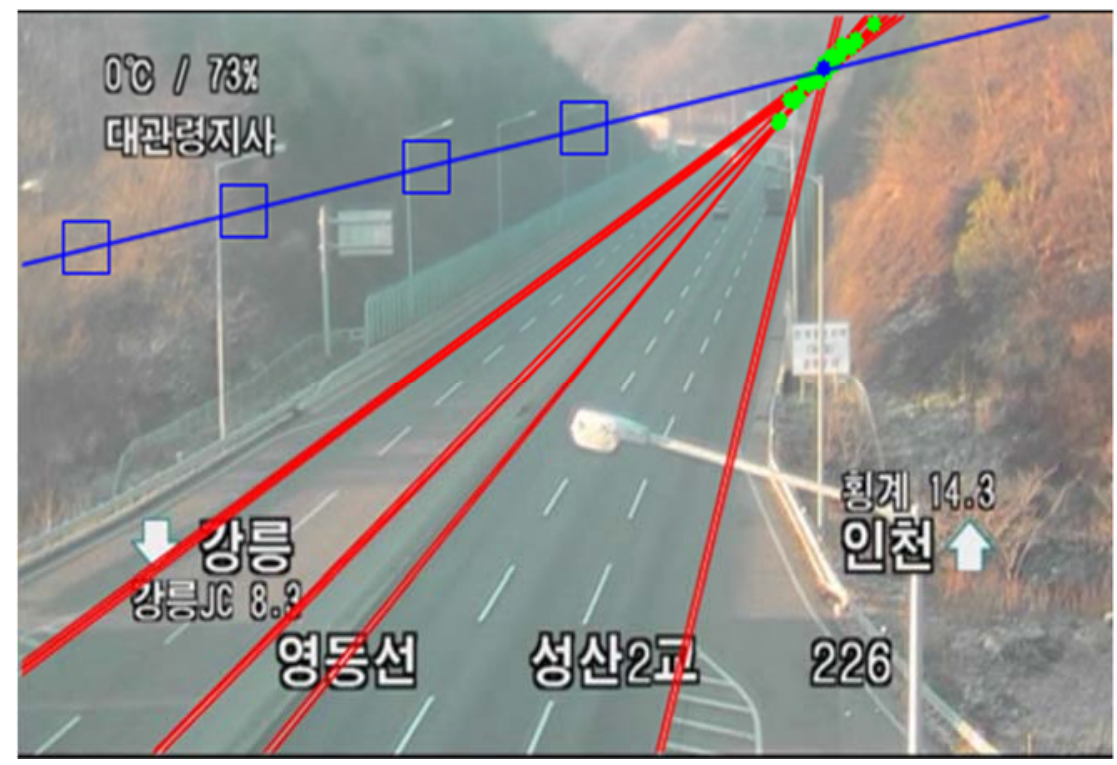

Figure 9 example of multiple ROI 


\section{Learning Model for Clustering Climate Change Patterns}

Section 4 presents a learning model for clustering climate change patterns using multiple ROls of CCTV images. First, we define the difference of image conversion values between multiple ROls as a pattern. Clustering groups are created hierarchically using the similarity function of data patterns. For sub-clusters of hierarchical clustering, rainfall values are assigned as label values. The similarity function is used to find the closest sub-cluster to the generated data pattern from a current CCTV image.

\subsection{Generation of Pattern Data}

In this section, we describe an algorithm that generates a ROI data pattern that can detect weather changes well using multiple ROls defined by Section 3. The set of multiple region of interest is $\left\{\mathrm{roi}_{1}, \mathrm{roi}_{2}, \boldsymbol{r o i}_{3}, \ldots, \mathrm{roi}_{n}\right\}$, where $\mathrm{n}$ is the total number of element of the ROI. The ROI data pattern is defined as a set of difference value between $r o i_{i}$ and $r o i_{j}$ because the difference value between ROls is well representative of weather change, and the $p t d_{k}$ is an element of the ROI data pattern. The $p t d_{k}$ is calculated by Equation (8).

$p t d_{k}=F_{\text {diff }}\left(p t d^{r o i_{i}}, p t d^{r o i_{j}}\right)=F_{\text {avr }}\left(p t d^{r o i_{i}}\right)-F_{\text {avr }}\left(p t d^{r o i_{i}}\right)$

(8), where $i$ and $j$ are a specific roi, $k$ is a specific ROI data pattern, and $F_{a v r}\left(p t d^{r o i_{i}}\right)$ is the average value of the pixels in the region of $r o i_{i}$.

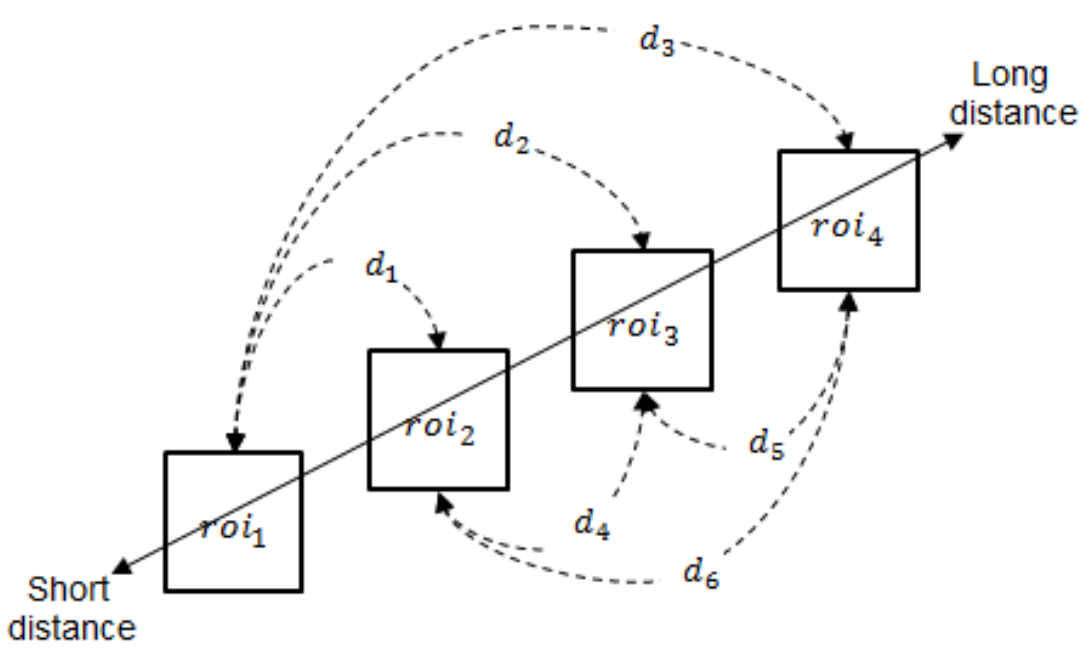

Figure 10 An example of a ROI data pattern

The number of elements in the ROI data pattern is the number of subsets having two elements for $\mathrm{n}$ ROIs. As shown in Fig. 10, if there are a set ROI with 4 elements, the number of subsets with two elements is 6 according to Eq. (9) such as $\left\{r i o_{1}, \mathrm{roi}_{2}\right\}_{1}\left\{\mathrm{rio}_{1}, \mathrm{roi}_{3}\right\}_{\text {, }}$ 
$\left\{\mathrm{rio}_{1}, \mathrm{roi}_{4}\right\},\left\{\mathrm{rio}_{2}, \mathrm{roi}_{3}\right\},\left\{\mathrm{rio}_{2}, \mathrm{roi}_{4}\right\},\left\{\mathrm{rio}_{3}, \mathrm{roi}_{4}\right\}$.

$$
\mathrm{C}(\mathrm{n}, \mathrm{r})=\frac{n !}{r !(n-r) !}
$$

, where $\mathrm{n}$ is the number of elements in the set ROI, and $r$ is the number of elements constituting one pair, in this case 2.

\subsection{Clustering of ROI Data Pattern}

The clustering of the ROI data pattern is to transform the past CCTV video data into a learning model of past rainfall changes. In this paper, we use hierarchical clustering algorithm to cluster pattern data. The hierarchical clustering algorithms is a "bottom up" approach: each observation starts in its own cluster, and pairs of clusters are merged as one moves up the hierarchy. The similarity function used in the hierarchical clustering algorithm is represented by the Euclidean distance formula, as shown in Equation (10).

Euclidean Distance $(q, p)=\sqrt{\sum_{i=0}^{n}\left(q_{i}-p_{i}\right)^{2}}$

where $\mathrm{n}$ is the number of pattern attributes

Definition (Hierarchical Cluster) Hierarchical cluster (HCR) consists of multiple sub-cluster $s c_{i}$, each sub-cluster $s c_{i}$ is a set of historical ROI data pattern. It is expressed as $H C R=$ $\left\{s c_{x} \mid, x\right.$ is from 1 to the number of groups $\}$. Let $F_{s}\left(p t d_{k}, p t d_{l}\right)$ be the similarity between any $p t d_{k}$ ROI data pattern of any sub-cluster $s c_{i}$ and any $p t d_{l}$ ROI data pattern of any sub-cluster $s c_{i}$. At this time, all data pattern of sub-cluster $s c_{i}$ satisfies the condition of always having higher degree of similarity than $F_{s}\left(p t d_{k}, p t d_{l}\right)$.

In Fig. 11, after converting the historical CCTV image into the ROI data pattern defined in 4.1, we constructed the hierarchical cluster using the ROI data pattern. The distance matrix (DM) computing all the ROI data patterns is calculated by equation (10). We defined the distance measurements between the clusters as "average connections" and performed hierarchical clustering with the DM as input. Fig. 11 shows an example of a hierarchical cluster composed of six sub-clusters, and is represented by a dendrogram for easy visual identification. As a result of the hierarchical clustering, each sub-cluster $s c_{i}$ can be classified into a set of finite pattern data having similar values. 


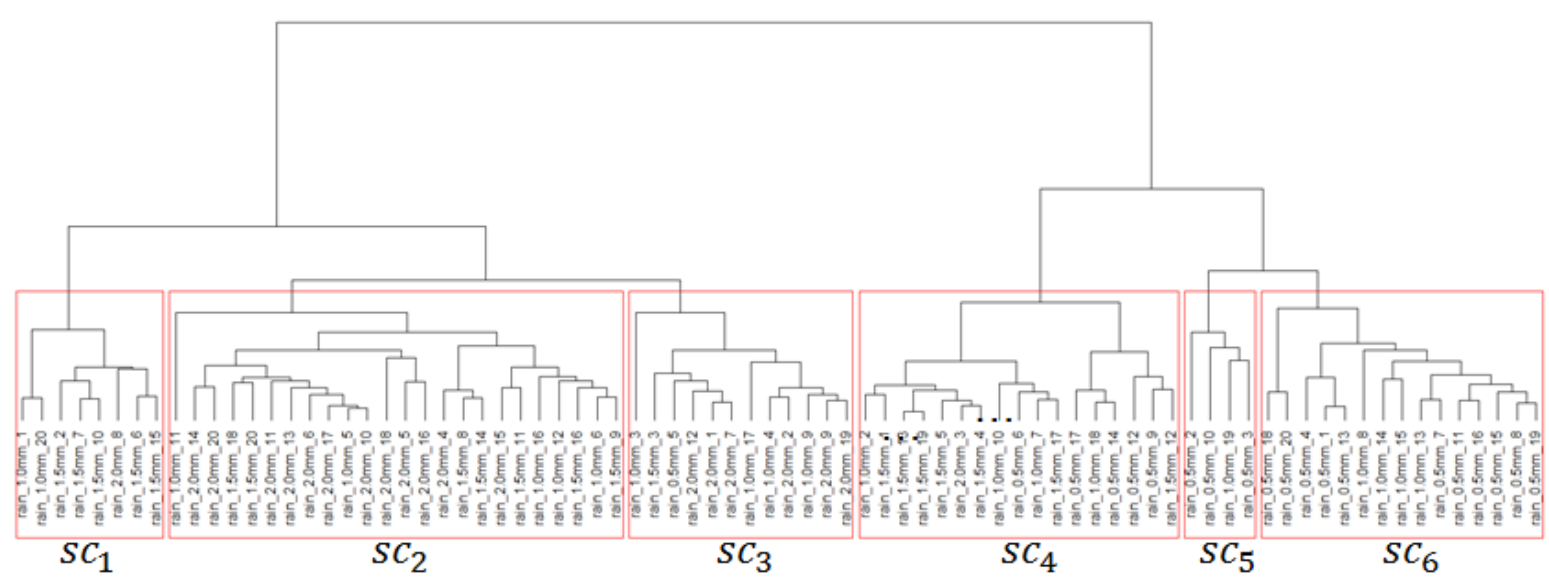

Figure 11 An example of clustering of ROI data pattern based on hierarchical clustering method

\subsection{Defining Weather information of Sub-Clusters}

This section describes how to assign representative ROI data patterns and weather information attributes to sub-clusters $s c_{i}$. The $s c_{i}$ has representative value as expressed in $<$ RPTD, WI $>$. The RPTD is $\left\{r d_{1}, r d_{2}, r d_{3}, \ldots, r d_{n}\right\}$, and $\mathrm{n}$ is the number of pattern data determined by equation (9). Equation (11) is a formula for calculating the element $d_{i n}$ of the set $p t d_{k}$.

$$
r d_{k}=\sum_{i=1}^{l} \frac{d_{i k}}{l}
$$

(11), where $d_{i k}$ is $k^{\text {th }}$ item of $p t d_{i}$

The representative precipitation of sub-cluster $s c_{i}$ is determined based on the frequency function. To prepare the data, we extract the weather data of the sub-cluster $s c_{i}$ and calculate the frequency of precipitation for the sub-cluster using the word counter algorithm. And then, the top two precipitation amounts with the highest frequency are then selected by outlier removal. The selected precipitation should be assigned a range of precipitation with start and end. Fig. 12 shows the order of assigning representative pattern and precipitation information to sub-cluster. 


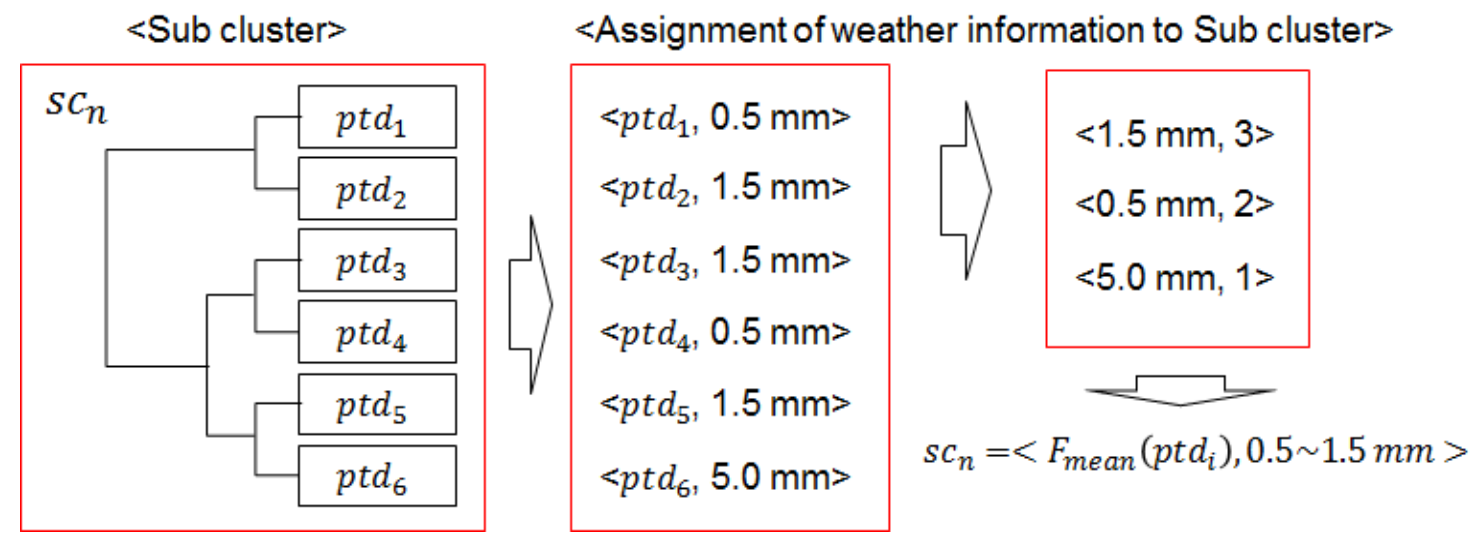

Figure 12 Example of assigning representative pattern and precipitation information to subcluster

\subsection{Clustering Model based Weather Information Production}

As described in table 2. Clustering model based weather information production algorithm consists of four steps:

Step 1 generates ROI data patterns from the big data of CCTV video and corresponds to lines 3 to 8 . Line 5 calculates the difference between the ROIs.

Step 2 is a logic for creating hierarchical clusters, from lines 9 to 15. On lines 11 and 12, the distance matrix is generated by measuring the similarity for all the ROI data patterns created in Step 1. Then, on line 15, the DM is input and a hierarchical cluster is created.

Step 3 assigns representative $\mathrm{ROI}$ data patterns and precipitation to sub-clusters of hierarchical clusters. On the line $18, \mathrm{ROI}$ data patterns corresponding to a specific subcluster are extracted, and on the lines 19 and 20, an average value of the ROI data patterns is calculated to generate a representative pattern value. Line 21 uses the frequency function to return the rainfall of the two highest frequencies. The sub-cluster has a representative value of these two pieces of information.

Step 4 is from line 24 to 28 , and returns the road weather information by finding the subcluster that is most similar to the ROI data pattern data of a given image. In the case of 25 line, the sub-clusters with the highest similarity are extracted in comparison with all the sub-clusters. In line 27 , the precipitation of the sub-clusters is assigned. Finally, the optimally approximated weather value found on line 28 is returned. 
Table 2. Clustering learning model based weather information production

Input: Historical Difference Graph $H D G$, Current CCTV video $C V$, multiple ROI ROI

Output: Weather Condition WC

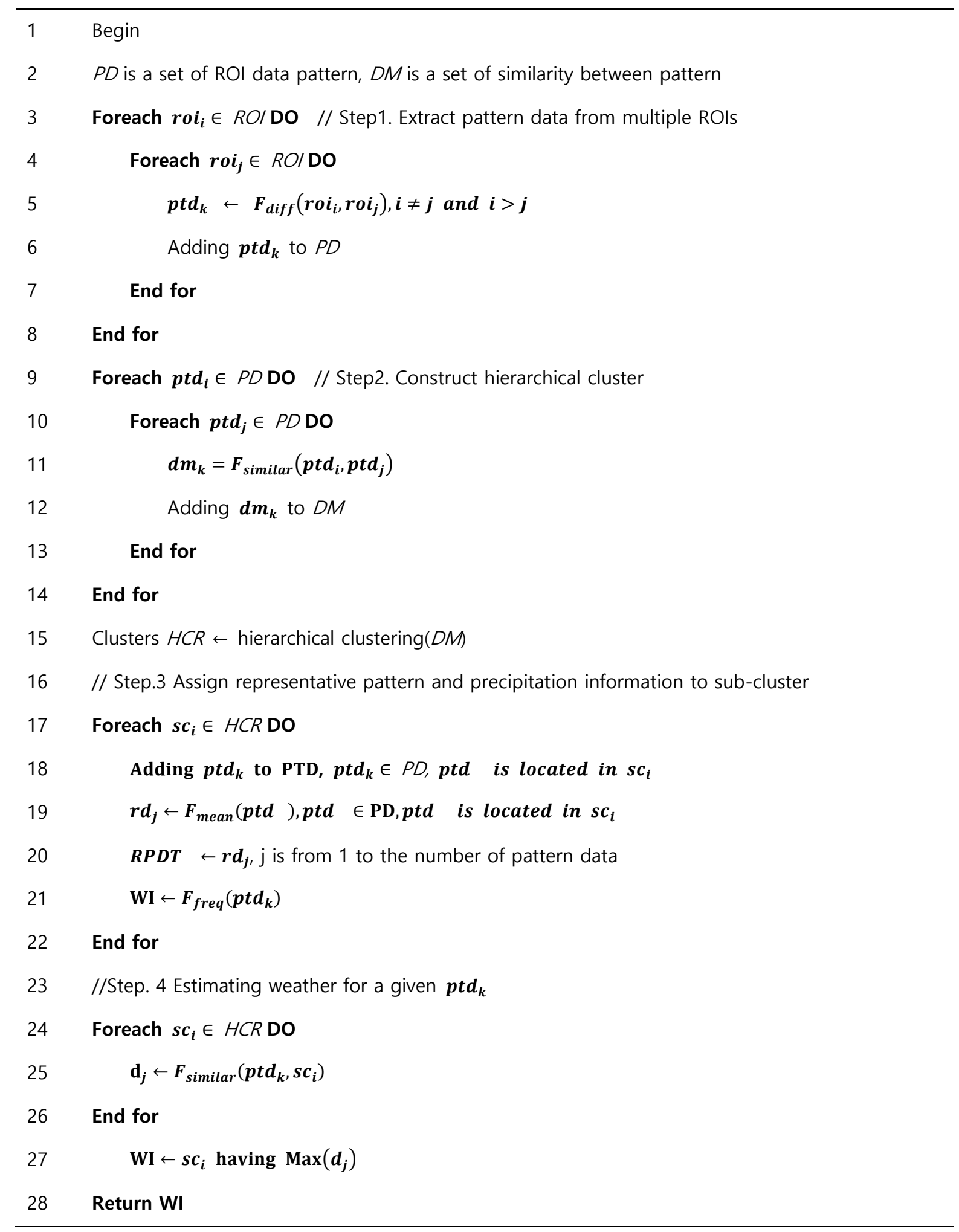




\section{Evaluation}

In this section, we evaluate the effectiveness of the selection of background area in CCTV video and the optimal set of multiple ROls that can best represent distinctive data pattern for different CCTV images presented in Section 3. We evaluate the accuracy of weather estimation model based weather information production algorithm proposed in Section 4.

\subsection{Experiment Environment}

Two CCTVs, one fixed weather observation equipment, and one mobile weather observation equipment were used to verify the accuracy of producing weather information of the road section by analyzing big data of CCTV video. Actual CCTV images from road management companies are 200,000 still images at different times. The actual CCTV images would be collected from October 2016 to April 2017, near the Pyeongchang Olympic site in Yeongdong Expressway in Korea.

Stationary Weather Observation Equipment RWIS is usually installed at a fixed pole and has a high accuracy of meteorological observations because it uses a high precision sensor to measure rainfall. The weather observation equipment attached to the moving vehicle was used to collect an approximated rainfall measurement for the road section where no RWIS is installed. The amount of rainfall collected as meteorological observations was observed in from October 2017 to April 2017 as actual data.

\subsection{Scenarios}

In this experiment, we conducted two categories of experiments to verify the proposed clustering learning based weather estimation model. The first is an experiment on the background area of CCTV video and the selection of multiple regions of interest. The second is a verification experiment on the accuracy of meteorological information production based on clustering learning model. In order to carry out this experiment, the experiment was conducted by dividing the case where the stationary RWIS exists on the designated road section and the case where there is no stationary RWIS on the road section. This is because a clustering learning model for a road section without a fixed RWIS is required to apply the proposed algorithm to a real environment. If there is no stationary RWIS in the road section where the CCTV is installed, the stationary RWIS data of the nearest road section and the movable RWIS data are used as the rainfall estimation value of the corresponding road section. In Figure 13, zone A is the case with stationary RWIS and Zone B is without RWIS. 


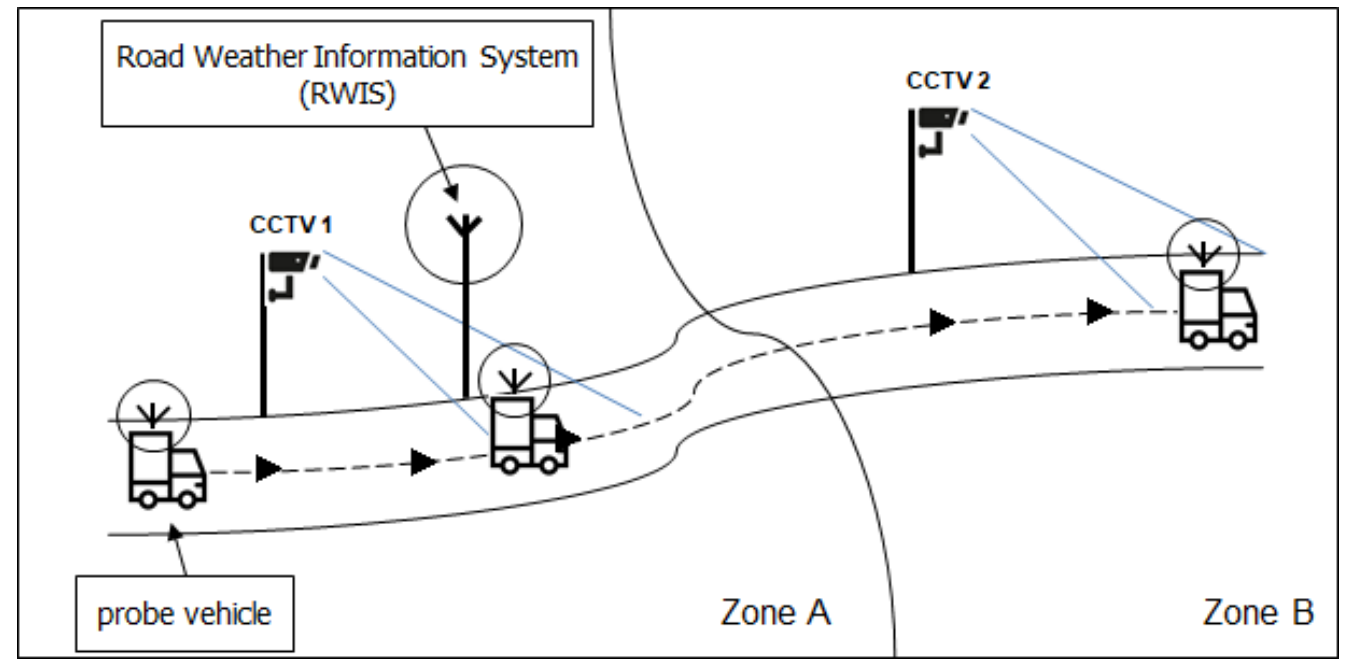

Figure 13 Experiment with stationary RWIS and mobile RWIS

\subsection{Finding Optimal ROI}

In this section, we show the results of experiments on the selection of the background area of CCTV video and the optimal selection of multiple ROIs

\subsubsection{Experiment on the selection of the background area of CCTV video}

In this experiment, CCTV images were transformed into HSV color spaces to extract useful features that appear according to weather changes and detected changes in brightness (Value) values in forests and roads. We analyzed the pattern conversion of the image conversion values in the roads and forest areas according to the weather, sunny, cloudy, $10 \mathrm{~mm}$, and $20 \mathrm{~mm}$. The analysis of the change in the HSV color space for the CCTV images revealed that the pattern based on the brightness value can best represent fine weather changes.

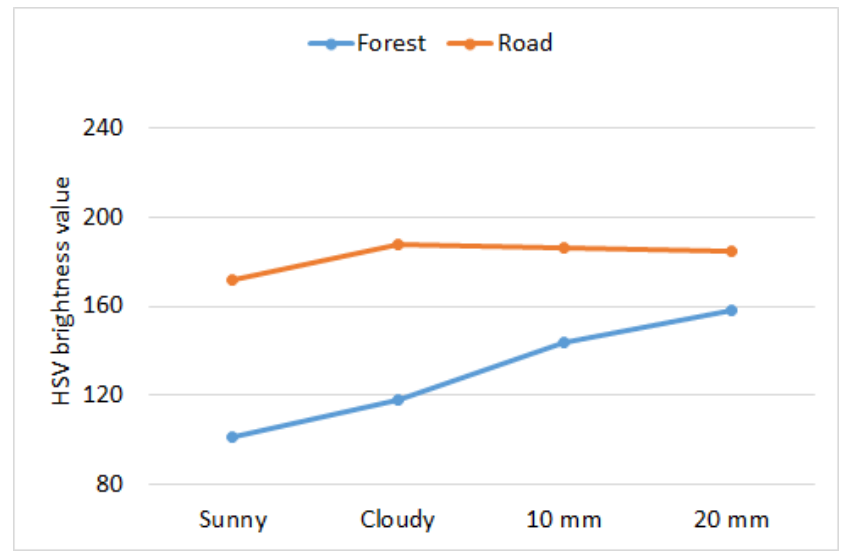

Figure 14 Comparison of brightness values for forest and road areas. HSV: hue-saturation- 


\section{brightness}

Fig. 14 shows that the pattern changes in the brightness value according to the weather changes in road and forest. It shows how it differs from area to area. Fig. 14, the brightness value is generally lower in the forest area than in the road area. The road area shows a gradual change of brightness value between sunny, cloudy, and rainfall. On the other hand, the forest area has a large variation of brightness value in rainfall compared to sunny. In addition, the road area does not show a change in brightness value when the rainfall changes from $10 \mathrm{~mm}$ to $20 \mathrm{~mm}$. However, the forest area shows a clear difference in the brightness value even when such rainfall changes.

\subsubsection{Experiment on size and spacing of $\mathrm{ROI}$}

In this experiment, a comparison experiment was carried out by constructing various sizes and intervals of ROI in CCTV images. Experiments to find the optimal ROI size in CCTV video have been performed with fixed spacing and number of ROls between ROls. The experiment in Fig. 15 shows that the interval between ROls is 150 pixels and the number of ROIs is fixed to 4 . The $d i$ in the $x$-axis of Figure 15 represents the difference in brightness value between ROIs. For example, D1 represents the difference between the closest ROls. Fig. 15 shows that the change of brightness value is greatest when the ROI size is $25 \times 25$. These experiments were also applied for different ROI intervals of 120,150 , and 180 pixels.

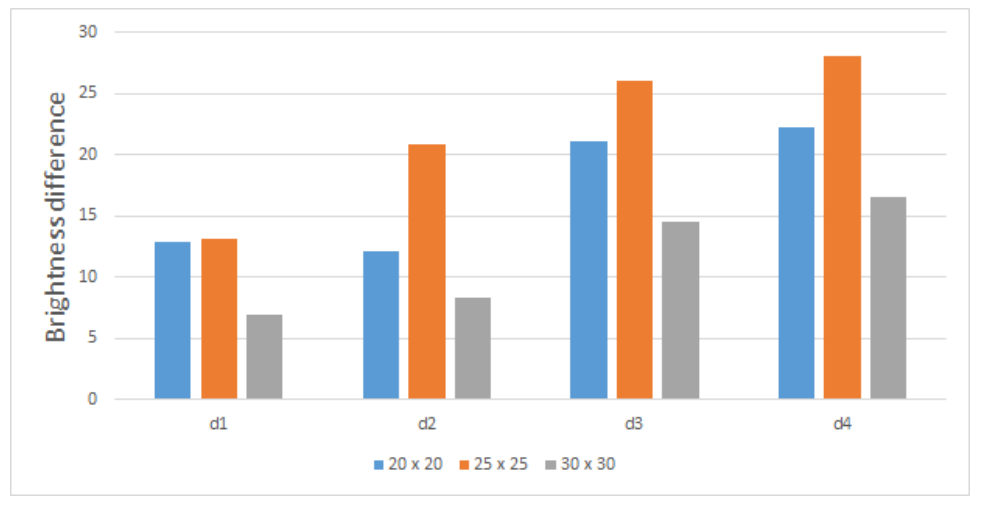

\section{Figure 15 Experiment of change of brightness value for difference ROI size}

The graph shown in Fig. 15 shows the distance between two ROIs on the $x$-axis. The larger the number of $n$, the farther the distance between ROls is, as shown in Fig. 10. The function to calculate the $\mathrm{Y}$ value corresponding to $d n$ is as follows. $\mathrm{f}(n)=$ $\mid B T$ of $r o i_{n-1}-B T$ of $r o i_{n} \mid$, where BT is brightness. This function is to obtain the 
brightness difference value between two ROIs. The large difference in brightness means that the characteristics of the weather are well represented by the ROI distance. Fig. 15 shows the results of experiments on $d 1, d 2, d 3$, and $d 4$ with ROIs of 20x20, $25 \times 25$, and $30 \times 30$. As a result of the experiment, the best value of the brightness difference is $25 \times 25$. It is appropriate to determine the size of the ROI area of $25 \times 25$ in order to find the characteristics of the image according to the short distance / long distance ROI according to the weather condition..

Experiments were performed to compare the brightness patterns of the different weather conditions according to ROI distance intervals in order to select the optimum interval between multiple ROIs in CCTV images. Taking into account the size of the forest area, the pixels were fixed at 120,150, and 180 on the target image data for the experiment. Table 3 shows the brightness value results when the ROI distance interval was varied for each weather condition. The first row of Table 3 shows the brightness values of the four ROls R1, R2, R3, and R4 in the sunny condition with a 120-pixel interval, which are $92.38,89.46,94.33$, and 103.24 , respectively. To summarize the contents of the table: in sunny weather, the difference was greater when the ROI interval was 180 pixels. In the case of cloudy and rainy days, however, the difference in the values was not significant as the interval increased to 150 pixels or more. From the experiment results in Table 3, it was concluded that the ROI interval should not exceed a maximum of 150 pixels when precipitation is determined.

Table 3 Brightness values according to ROI interval for each weather condition

\begin{tabular}{|c|c|c|c|c|c|}
\hline $\begin{array}{l}\text { Weather } \\
\text { Condition }\end{array}$ & $\begin{array}{c}\text { Distance } \\
\text { between } \\
\text { ROIs }\end{array}$ & $\mathrm{R} 1$ & $\mathrm{R} 2$ & R3 & $\mathrm{R} 4$ \\
\hline \multirow{3}{*}{ sunny } & 120 pixel & 92.38 & 89.46 & 94.33 & 103.24 \\
\hline & 150 pixel & 86.91 & 90.43 & 96.19 & 97.25 \\
\hline & 180 pixel & 86.76 & 91.53 & 103.32 & 109.62 \\
\hline \multirow{3}{*}{ cloudy } & 120 pixel & 103.49 & 108.47 & 119.11 & 120.11 \\
\hline & 150 pixel & 101.95 & 115.13 & 122.77 & 127.99 \\
\hline & 180 pixel & 104.51 & 109.14 & 119.99 & 128.43 \\
\hline \multirow{3}{*}{$\begin{array}{l}\text { rainy } \\
10 \mathrm{~mm}\end{array}$} & 120 pixel & 126.04 & 126.10 & 132.54 & 142.10 \\
\hline & 150 pixel & 116.46 & 125.97 & 142.66 & 158.51 \\
\hline & 180 pixel & 117.46 & 134.61 & 141.87 & 162.40 \\
\hline \multirow{3}{*}{$\begin{array}{l}\text { rainy } \\
20 \mathrm{~mm}\end{array}$} & 120 pixel & 123.45 & 130.30 & 145.05 & 157.13 \\
\hline & 150 pixel & 119.97 & 134.45 & 154.52 & 172.92 \\
\hline & 180 pixel & 121.47 & 139.53 & 155.49 & 175.02 \\
\hline
\end{tabular}




\subsection{Accuracy of the Clustering Learning Model}

Experiment1 (Zone A) From April 17th to 19th, 2017, we performed an experiment to compare the rainfall calculated by the clustering learning model with the stationary RWIS meteorological observations using the CCTV video data of the rainy highway. The location of the CCTV used in the experiment was selected very close to stationary RWIS. The rainfall images used for clustering were 100,000 images for $0.5 \mathrm{~mm}, 1.0 \mathrm{~mm}, 1.5 \mathrm{~mm}$, and $2.0 \mathrm{~mm}$. To avoid overfitting of clustering learning, 80 samples were randomly sampled for 100,000 images. The data used for the estimation of the rainfall amount for a given CCTV image was selected randomly from 40 images. The rows in Fig. 16 show that there are four image types used in the experiment verification and the results are in the number of images in the columns. As a result of Fig. 16, the proposed clustering learning model has an accuracy of about $87.5 \%$.

\begin{tabular}{|l|c|c|c|c|}
\hline Rainfall & $0.5 \mathrm{~mm}$ & $1.0 \mathrm{~mm}$ & $1.5 \mathrm{~mm}$ & $2.0 \mathrm{~mm}$ \\
\hline $0.5 \mathrm{~mm}$ & 10 & 0 & 0 & 0 \\
\hline $1.0 \mathrm{~mm}$ & 0 & 10 & 0 & 0 \\
\hline $1.5 \mathrm{~mm}$ & 0 & 0 & 10 & 0 \\
\hline $2.0 \mathrm{~mm}$ & 0 & 0 & 5 & 5 \\
\hline
\end{tabular}

Figure 16 Experimental results of weather estimation in difference region

Experiment2 (Zone A) The second experiment is the same as the first experiment, except that the target location is different. We used the mobile RWIS meteorological data to verify the meteorological data of the second experiment. Clustering learning was performed on the spot where rainfall occurred from October 14 to 16, 2016, and CCTV images of the same time zone were verified. Fig. 17 (a) shows that the accuracy is very high at $97.5 \%$. This is because the variability of the image data used for learning and verification is poor.

Experiment3 (Zone B) In the third experiment, the learning was performed in the same place, and then the verification experiment was performed for different time zones in the same place. After learning from the data collected between October 14 and 16, 2016 for the same place used for the second experiment, a verification experiment was conducted on April 17, 2017. The experimental results show that the accuracy drops to $80.0 \%$ as shown 
in Fig. 17 (b). This is because the degree of variability of the data used in clustering learning affects accuracy. Finally, in order to increase the accuracy by clustering learning, it is necessary to accumulate learning using various kinds of CCTV videos for various weather changes.

\begin{tabular}{|c|c|c|c|c|}
\hline Rainfall & $0.5 \mathrm{~mm}$ & $1.0 \mathrm{~mm}$ & $1.5 \mathrm{~mm}$ & $2.0 \mathrm{~mm}$ \\
\hline $0.5 \mathrm{~mm}$ & 10 & 0 & 0 & 0 \\
\hline $1.0 \mathrm{~mm}$ & 0 & 10 & 0 & 0 \\
\hline $1.5 \mathrm{~mm}$ & 0 & 1 & 10 & 0 \\
\hline $2.0 \mathrm{~mm}$ & 0 & 0 & 0 & 10 \\
\hline
\end{tabular}

\begin{tabular}{|c|c|c|c|}
\hline Rainfall & $0.5 \mathrm{~mm}$ & $1.0 \mathrm{~mm}$ & $1.5 \mathrm{~mm}$ \\
\hline $0.5 \mathrm{~mm}$ & 10 & 0 & 0 \\
\hline $1.0 \mathrm{~mm}$ & 0 & 6 & 4 \\
\hline
\end{tabular}

(a)The result of experiment 2

(b) The result of experiment 3

Figure 17 Experiment with CCTV images in same places and other time zone

Experimental Summary In order to evaluate clustering learning model for estimating road weather information from CCTV video big data, we performed accuracy test of the method of generating weather information based on the clustered learning proposed in this paper. Experiment 1 and Experiment 2 are the results of testing the accuracy of different points. Experiment 3 is the result of experiment on CCTV images at different time for the same point. Experiment 3 was a test for only two cases of $0.5 \mathrm{~mm}$ and $1.0 \mathrm{~mm}$ precipitation. As shown in Fig. 18, for all $5 \mathrm{~mm}$ precipitation, all three experiments show 100\% accuracy. For $1.0 \mathrm{~mm}$ precipitation, Experiment 1 and Experiment 2 had 100\% accuracy, whereas Experiment 3 had about 60\% accuracy. In Experiment 3, although the same place as Experiment 2 was tested, the reason for the low accuracy was that the time gap between the training data and the test data was about 7 months. Although the images obtained from CCTV are similar rainfall, it can be seen that they may appear differently at different times. For the $1.5 \mathrm{~mm}$ precipitation, both Experiment 1 and Experiment 2 showed an accuracy of $95 \%$ or more. For the $2.0 \mathrm{~mm}$ precipitation, the accuracy was significantly reduced to $50 \%$ of Experiment 1. In Experiment 1, the accuracy was reduced by about $50 \%$ because the $2.0 \mathrm{~mm}$ precipitation was classified as $1.5 \mathrm{~mm}$ precipitation. Although we may have doubts about the effectiveness of $50 \%$ accuracy, it is considered to be of sufficient value as road hazard weather information for road drivers because of slight change of 0.5 $\mathrm{mm}$ rainfall. 


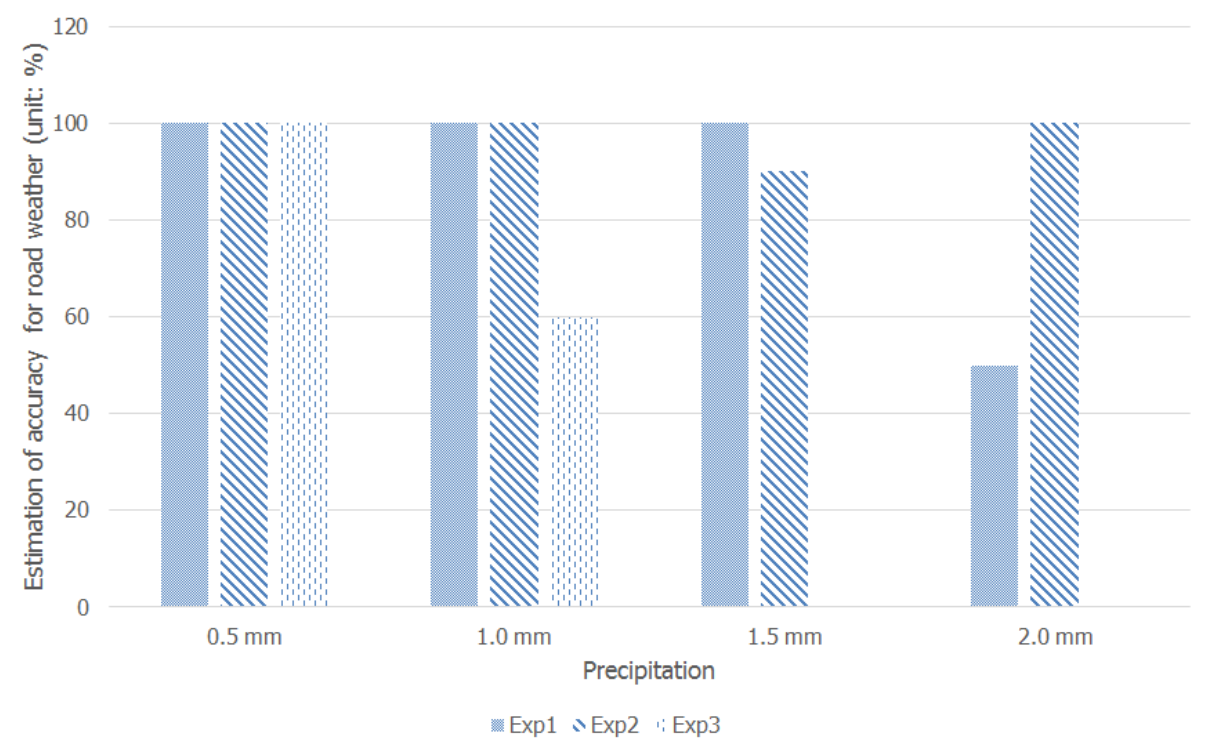

Figure 18 Analyzing the accuracy of different rainfall, different time zones

\section{Related Work}

Due to frequent extreme weather event, meteorological information is a field of common interest worldwide. Visualization and forecasting analysis of weather information [21] is being studied by using the latest technologies $[9,10,11,12,22]$ based on loT, Big data analytics, and cloud computing. Recently, road hazard weather information, which is a combination of road and weather information, is an issue. The reason is that road hazard weather information is very important information that directly affects traffic accidents.

Previous research on road hazard weather information has emphasized the importance of identifying and providing dangerous roads to vehicle drivers in advance to prevent traffic accidents $[23,24,25]$. In order to collect road weather information, there have been previous studies $[26,27,28,29]$ that produce road weather information using roadside CCTV images instead of costly fixed RWIS. However, previous studies have focused only on state information of road weather such as snow, fog, and rain on the road. No learning model has been attempted to estimate the amount of rainfall to be addressed in this paper.

The study of extracting road weather information from CCTV images can be tried to use whole image or specific region of image according to ROI selection method. When the entire image is selected as the ROI, there is a problem that the accuracy varies depending on the components of the image such as roads, forests, sky [4]. The existing research method did not distinguish whether the specific area which shows the change of weather 
information in CCTV images depends on the forest area or roads area.

In order to extract the road weather information from CCTV images, the conversion values such as RGB average value, temperature and humidity information, HSV value and edge pixel amount $[30,31,32]$ have been used in the previous studies to extract the CCTV image from the CCTV video. However, these models have the disadvantage that they are not applicable to real environments with threshold based methods. In addition, since the existing methods are the analysis using an image characteristic value of a corresponding region by simply selecting an ROI, it is difficult to find the weather change in CCTV images.

In this paper, we propose a method to define the difference patterns between the multiple ROls as a ROI data pattern for the corresponding image. In this paper, we propose a non-deterministic clustering learning model that can classify ROI data patterns according to rainfall variation into clusters. The non-deterministic clustering learning model proposed in this paper has the advantage of providing a robust clustering learning model in which the amount of data learning determines the accuracy.

\section{Conclusion}

It is shown in this paper that it is possible to produce road hazard weather information for sustainability of road safety driving and safety management by simply analyzing CCTV images of roadside instead of costly RWIS. Experiments have confirmed that existing CCTVs on roads can be able to sufficiently and effectively function as loT devices capable of producing road hazard weather information in addition to simple road control.

We have proposed a method to select multiple ROls that best show changes in CCTV images caused by weather changes. In order to produce road weather information, the difference of image conversion value between multiple ROls of a specific CCTV image is defined as a pattern for the corresponding CCTV image. We have proposed a clustering learning model that hierarchically clusters these CCTV image patterns and matches precipitation with sub-clusters' labels.

We have found that selecting multiple ROls that can best represent fine weather changes is a key success factor in solving the problem of generating road weather information by CCTV image data analysis. The automatic selection method of multiple ROI is implemented by using the Watershed algorithm to determine the detection target areas such as forest and roads through image segmentation. In order to select optimal multiple ROI, experiments were performed on real images to best show the image transformation 
patterns of horizontal, vertical and roads of CCTV screen. As a result of the experiment, selecting multiple ROls based on the roads was the most effective. It was verified by experiments using real images that the optimal size of the ROI used for multiple ROls is $25 \times 25$ and that the optimum spacing between the ROls is over 150 pixels.

The most important contribution of this paper is to define the difference of the image conversion value between multiple ROls as a ROI data pattern of the corresponding image, and then to make the learning possible by clustering patterns of past CCTV images hierarchically. The algorithm to identify the road hazard weather information using the generated hierarchical clustering is composed of 4 steps as follows: 1) Generate the difference pattern for the image conversion value between the selected multiple ROIs, 2) The difference patterns between multiple ROIs generated for past CCTV images are clustered hierarchically using the similarity function. 3) For each sub-cluster in the hierarchical clustered tree, the meteorological observations are assigned as label values. 4) The sub-cluster closest to the change pattern between multiple ROIs for the new CCTV image is selected using the similarity function to generate road weather information. As a result of the experiment using the real image, it proved that the clustered learning model generated in the same region has more than $80 \%$ accuracy. As the number of past weather observations and CCTV images in the same area increases, the accuracy of the generated weather information can be further improved.

It is expected to be compared with the experiment using deep learning method for CCTV images expressing minute weather changes such as rainfall. In this paper, it is necessary to compare the robustness of the clustering learning model of ROI data patterns with deep earning. 


\section{Reference}

[1] Yang, Hun-Jun, et al. "Classification algorithm for road surface condition." International Journal of Computer Science and Network Security (IJCSNS) 14.1 (2014): 1.

[2] Qian, Yiming, Emilio J. Almazan, and James H. Elder. "Evaluating features and classifiers for road weather condition analysis." Image Processing (ICIP), 2016 IEEE International Conference on. IEEE, 2016.

[3] Andrey, Jean, et al. "Weather as a chronic hazard for road transportation in Canadian cities." Natural Hazards 28.2 (2003): 319-343.

[4] Lee, Jiwan, et al. "Extraction of weather information on road using CCTV video." Big Data and Smart Computing (BigComp), 2016 International Conference on. IEEE, 2016.

[5] Toshev, Alexander, and Christian Szegedy. "Deeppose: Human pose estimation via deep neural networks." Proceedings of the IEEE Conference on Computer Vision and Pattern Recognition. 2014. [6] Cao, Zhe, et al. "Realtime multi-person 2d pose estimation using part affinity fields." arXiv preprint arXiv:1611.08050 (2016).

[7] Donahue, Jeffrey, et al. "Long-term recurrent convolutional networks for visual recognition and description." Proceedings of the IEEE conference on computer vision and pattern recognition. 2015. [8] He, Kaiming, et al. "Spatial pyramid pooling in deep convolutional networks for visual recognition." IEEE transactions on pattern analysis and machine intelligence 37.9 (2015): 1904-1916. [9] Farahani, Bahar, et al. "Towards fog-driven loT eHealth: promises and challenges of IoT in medicine and healthcare." Future Generation Computer Systems (2017).

[10] Ryu, Minwoo, et al. "Design and implementation of a connected farm for smart farming system." SENSORS, 2015 IEEE. IEEE, 2015.

[11] Breivold, Hongyu Pei, and Kristian Sandstrom. "Internet of Things for Industrial Automation-Challenges and Technical Solutions." Data Science and Data Intensive Systems (DSDIS), 2015 IEEE International Conference on. IEEE, 2015.

[12] Chapman, L., et al. "Winter road maintenance and the internet of things." Proceedings of the 17th International Road Weather Conference. Vol. 18. 2014.

[13] Lee, Jong-Won, and Jong-Ho Nang. "Multiple Region-of-Interest Based Image Retrieval Method." Journal of KIISE: Computer Systems and Theory 37.5 (2010): 314-318.

[14] Cheong, Cha-Keon. "A high speed road lane detection based on optimal extraction of ROI-LB." Journal of Broadcast Engineering 14.2 (2009): 253-264.

[15] OpenCV, http://opencv.

[16] Ivanovici, Mihai, Noël Richard, and Dietrich Paulus. "Color image segmentation." advanced color image processing and analysis. Springer New York, 2013. 219-277. 
[17] Hough_transform, https://en.wikipedia.org/wiki/Hough_transform

[18] Mukhopadhyay, Priyanka, and Bidyut B. Chaudhuri. "A survey of Hough Transform." Pattern Recognition 48.3 (2015): 993-1010.

[19] Duda, Richard O., and Peter E. Hart. "Use of the Hough transformation to detect lines and curves in pictures." Communications of the ACM 15.1 (1972): 11-15.

[20] Bilmes, Jeff A. "A gentle tutorial of the EM algorithm and its application to parameter estimation for Gaussian mixture and hidden Markov models." International Computer Science Institute 4.510 (1998): 126.

[21] Chang, Victor. "Towards data analysis for weather cloud computing." Knowledge-Based Systems 127 (2017): 29-45.

[22] Chang, Victor. "An overview, examples, and impacts offered by Emerging Services and Analytics in Cloud Computing virtual reality." Neural Computing and Applications (2017): 1-14.

[23] Shibata, Keiji, et al. "Detection of road surface conditions in winter using road surveillance cameras at daytime, night-time and twilight." International Journal of Computer Science and Network Security (IJCSNS) 14.11 (2014): 21.

[24] Raj, Arjun, Dilip Krishna, and Kumar Shantanu. "Vision based road surface detection for automotive systems." Applied Electronics (AE), 2012 International Conference on. IEEE, 2012.

[25] Tanaka, Y., et al. "A study on low-cost hazard detection system using CCTV image." 11th ITS Symposium. 2012.

[26] Shibata, Keiji, et al. "Distinction of wet road surface condition at night using texture features." Electronics and Communications in Japan 97.6 (2014): 51-57.

[27] Jonsson, Patrik. "Classification of road conditions: From camera images and weather data." Computational Intelligence for Measurement Systems and Applications (CIMSA), 2011 IEEE International Conference on. IEEE, 2011.

[28] Jokela, Maria, Matti Kutila, and Long Le. "Road condition monitoring system based on a stereo camera." Intelligent Computer Communication and Processing, 2009. ICCP 2009. IEEE 5th International Conference on. IEEE, 2009.

[29] Park, Beung-Raul, Sung Namkoong, and Joong-Tae Lim. "Development of the Road Weather Detection Algorithm on CCTV Video Images using Double Decision Trees." The KIPS Transactions: PartB 14.6 (2007): 445-452.

[30] Kim, Bong-Geun, In-Su Jang, and Gwang Lee. "Real-time Road-Visibility Measurement Using CCTV Camera." Journal of Korean Society of Transportation 29.4 (2011): 125-138.

[31] Jang, Tae-Woo, Yong-Tae Shin, and Jong-Bae Kim. "A study on the object extraction and tracking system for intelligent surveillance." The Journal of Korean Institute of Communications and 
Information Sciences 38.7 (2013): 589-595.

[32] Canny, John. "A computational approach to edge detection." IEEE Transactions on pattern analysis and machine intelligence 6 (1986): 679-698. 